\title{
Application of weighted total least squares based on intensity value in plane fitting of point clouds
}

\author{
Guihua Cang ${ }^{1,2}$, Mingfeng $\mathrm{Li}^{2}$, Jiangxia Ou ${ }^{2}$, Ling Zhu ${ }^{3,4}$ \\ ${ }^{1}$ School of Earth Sciences and Engineering, Hohai University, Nanjing, China \\ 2 Department of Geomatics Engineering, Nanjing University of Technology, Nanjing, China \\ ${ }^{3}$ Beijing University of civil engineering and architecture, Beijing, China \\ ${ }^{4}$ Key Laboratory of Precise Engineering and Industry surveying, National Administration of Surveying, Mapping and \\ Geoinformation, Beijing, China
}

\begin{abstract}
Aiming at the condition that each point in point clouds has different positional accuracy, a new method for fitting plane to point clouds is proposed. The method is based on weighted total least squares (WTLS). According to the effect of intensity of each point on positional accuracy and the structure of coefficient matrix, the weight matrix was defined. In order to verify the applicability of the proposed method, analytical experiments based on instant data of different materials were done, and comparisons between the method and traditional methods, such as least squares (LS) method and total least squares (TLS) method were implemented. The results show that the method is suitable for fitting plane to point clouds with good homogeneity, and more accurate parameters solution can be obtained as compared with TLS and LS. As for point clouds with poor homogeneity, the proposed method is unsuitable for the same operational use, while TLS is the most effective method.
\end{abstract}

Keywords - point clouds, weighted total least-squares, intensity value, weight matrix, plane fitting

\section{INTRODUCTION}

As for a new Geo-Informatics acquisition technology, terrestrial laser scanning technology is especially valuable for its rapid capturing of large amounts of data with high accuracy in the order of millimeters. It is now widely used in numerous areas. One of the main applications of terrestrial laser scanning technology is visualization, modeling and monitoring of manmade structures such as buildings. In the scanning scenery, there are many planar features which can be used for data reduction and point clouds registration. Therefore, the planar feature extraction has become an important task in building model reconstruction from point clouds data, and the extraction accuracy is directly related to the accuracy of its postprocessing applications.

The least squares (LS) method is commonly used for plane fitting. Every plane parameter estimation problem gives rise to an over-determined set of linear equation $A X=Y$. There is an underlying assumption that all the errors are confined to the observation vector $Y$. Unfortunately, this assumption is frequently unrealistic; instrument errors, human errors, sampling errors may make the coefficient matrix $A$ inaccurate. For solving the problem of errors-in-variables (EIV) model, Golub et al. (1980) first proposed the total least squares (TLS) method, considering the random errors and gross errors may exist both in the observation vector and the coefficient matrix. But for TLS method, it is assumed that the coefficient matrix and observation vector are independent and are equal variance. This assumption is not suitable for point cloud data since the accuracy of each point is different. The weighted total least squares (WTLS) method was developed by Markovsky et al. (2006) to solve the problem of unequal precision estimation. Schaffrin et al. (2008) improved the WTLS method, and introduced some design techniques of the weight matrix of the coefficient matrix and the algorithm steps.

In this paper a weighted total least squares method based on the intensity value of each point of the scanning point cloud is proposed. According to the structural characteristic of the coefficient matrix, a reasonable weight matrix was designed. The designated weight matrix can fix the constant element and adjust the data element simultaneously. At the same time, based on the effect of point intensity on its positional precision, each point fitting weight was determined by its intensity. At last, the effectiveness of the algorithm was studied through sample data.

\section{FUNCTIONAL MODELS AND METHODS}

After data preprocessing, a set of 3D coordinates $\left(X_{i}, Y_{i}, Z_{i}\right)(i=1,2, \cdots, n)$ of each point can be given. We assume that the equation of a plane can be expressed as in Eq. (1).

$$
Z_{i}=a X_{i}+b Y_{i}+c \quad(i=1,2, \cdots, n)
$$

where $n$ is the number of points, $a 、 b 、 c$ are the planar parameters. (2).

Eq. (1) can be written in matrix form, expressed as in Eq.

where

$$
Y=A X
$$

$$
\underset{n \times 1}{Y}=\left[\begin{array}{c}
Z_{1} \\
Z_{2} \\
\vdots \\
Z_{n}
\end{array}\right], \quad \underset{n \times 3}{A}=\left[\begin{array}{ccc}
X_{1} & Y_{1} & 1 \\
X_{2} & Y_{2} & 1 \\
\vdots & \vdots & 1 \\
X_{n} & Y_{n} & 1
\end{array}\right], \quad \underset{3 \times 1}{X}=\left[\begin{array}{c}
a \\
b \\
c
\end{array}\right]
$$

where $Y$ is the $n \times 1$ observation vector, $A$ is the $n \times 3$ coefficient matrix, $X$ is the (unknown ) $3 \times 1$ parameter vector. 
Assuming that the errors are in both of the observation vector and the coefficient matrix, the EIV model can be defined by functional relationship [3]:

$$
\begin{gathered}
Y-e_{Y}=\left(A-E_{A}\right) X \\
{\left[\begin{array}{c}
e_{Y} \\
e_{A}
\end{array}\right]=\left[\begin{array}{c}
e_{Y} \\
\operatorname{vec}\left(E_{A}\right)
\end{array}\right] \sim\left(\left[\begin{array}{l}
0 \\
0
\end{array}\right], \sigma_{0}^{2}\left[\begin{array}{cc}
Q_{Y} & 0 \\
0 & Q_{A}
\end{array}\right]\right)}
\end{gathered}
$$

where $e_{Y}$ is the respective $n \times 1$ vector of observation noise, $E_{A}$ is the $n \times 3$ matrix of random noise, "vec" denotes the operator that stacks one column of a matrix underneath the previous one, $Q_{Y}=P_{Y}^{-1}$ and $Q_{A}=P_{A}^{-1}$ are the symmetric and non-negative-definite cofactor matrix of $e_{Y}$ and $E_{A}$, respectively, and $\sigma_{0}^{2}$ is an unknown variance component.

According to the designs of $e_{Y}$ and the weight matrix of coefficient matrix, different error model is established, and the corresponding estimation method is used to obtain the planar parameters.

\section{Case $1 e_{A} \equiv 0$}

In this case only the errors in the observation vector are considered, then Eq. (4) can be converted to the classical Gauss-Markov (G-M) model which is defined as follows:

$$
Y=A X-e_{Y}, e_{Y} \sim N\left(0, \sigma_{0}^{2} P_{Y}^{-1}\right)
$$

It is well known that the LS method is used to solve Eq. (6) with the conditions of estimation criterion in the sense that:

$$
e_{Y}^{T} P_{Y} e_{Y}+e_{A}^{T} P_{A} e_{A}=\min
$$

And $\sigma_{0}^{2}$ can be calculated as follows:

$$
\sigma_{0}^{2}=\frac{e_{Y}^{T} P_{Y} e_{Y}}{n-3}
$$

Case $2 e_{A} \neq 0$, but $Q_{Y}=I_{n}$ and $Q_{A}=I_{n \cdot m}$

In this case the errors both in the observation vector and the coefficient matrix are considered, but under the assuming that it is a homoscedastic case where all variances are equal and all co-variances is zero. The TLS method is used to estimate the planar parameters with the conditions of estimation criterion in the sense that:

$$
e_{Y}^{T} e_{Y}+e_{A}^{T} e_{A}=\min
$$

And $\sigma_{0}^{2}$ can be calculated as follows:

$$
\sigma_{0}^{2}=\frac{e_{Y}^{T} e_{Y}+e_{A}^{T} e_{A}}{n-3}
$$

Case $3 e_{A} \neq 0$, and both $Q_{Y}$ and $Q_{A}$ are not unit matrix

In this case the data have been observed with different precision, and the observations are heteroscedastic and correlated. According to the data characteristic and the structural of the coefficient matrix, the weight matrix $P_{0}$, $P_{X}$ and $P_{Y}$ are designed. $P_{0}$ is the $3 \times 3$ vector, denoting the column vector of coefficient matrix, $P_{X}$ is the $n \times n$ vector, denoting the weight matrix of the row vector of coefficient matrix. $P_{A}=\left(P_{0} \otimes P_{X}\right)$ is the weight matrix of coefficients matrix, where $\otimes$ is used to denote the Kronecker product. $P_{Y}$ is the weight matrix of observation vector $Y \cdot Q_{0} 、 Q_{X}$ and $Q_{Y}$ are the corresponding cofactor matrix of $P_{0} 、 P_{X}$ and $P_{Y}$ respectively, namely

$$
Q_{0}=P_{0}^{-1} \quad Q_{X}=P_{X}^{-1} \quad Q_{Y}=P_{Y}^{-1}
$$

From $Q_{0}$ and $Q_{X}$, we can obtain $Q_{A}$ and $P_{A}$ from Eq. (12).

$$
Q_{A}=P_{A}^{-1}=Q_{0} \otimes Q_{X}=P_{0}^{-1} \otimes P_{X}^{-1}
$$

The WTLS method is used to estimate the planar parameters with the conditions of estimation criterion in the sense that:

$$
e_{Y}^{T} p_{Y} e_{Y}+e_{A}^{T}\left(P_{0} \otimes P_{X}\right) e_{A}=\min
$$

And $\sigma_{0}^{2}$ can be calculated as follows:

$$
\sigma_{0}^{2}=\frac{e_{Y}^{T} p_{Y} e_{Y}+e_{A}^{T}\left(P_{0} \otimes P_{X}\right) e_{A}}{n-3}
$$

\section{THE ALOGRITHM OF WEIGHTED TOTAL LEAST SQUARES BASED ON INTENSITY VALUE}

\section{A. The determination of weight matrix}

We all know that each point of point clouds has different positional accuracy. When we fit a plane to point clouds, the fitting weight of each point is determined by its accuracy. The high the accuracy is, the larger the fitting weight is. This ensures the precision of fitting planar parameters. On the other hand, for the scanning point cloud, the effects of incidence angle on point intensity and its positional precision are significant. The point intensity values and its positional precisions decrease with increasing incidence angle [4]. So we can conclude the relationship between the point intensity value and its positional precision. The larger the intensity is, the higher its positional precision is, and so the higher its fitting weight is. Simultaneously considering the structural of the coefficient matrix, the weight matrix $P_{0} 、 P_{X}$ and $P_{Y}$ are designed as follow [3].

$$
\begin{aligned}
& \underset{3 \times 3}{P_{0}}=\left[\begin{array}{ccc}
1 & 0 & 0 \\
0 & 1 & 0 \\
0 & 0 & 0
\end{array}\right] \\
& \underset{\substack{X \times n \\
P_{X}}}{=P_{Y \times n}}=\left[\begin{array}{cccc}
E_{1} & 0 & \cdots & 0 \\
0 & E_{2} & \cdots & 0 \\
\vdots & \vdots & & \vdots \\
0 & 0 & \cdots & E_{n}
\end{array}\right]
\end{aligned}
$$

Here the third diagonal element of $P_{0}$ is zero, meaning that the third column of coefficient matrix is fixed and equal to 1 , while the other diagonal elements of $P_{0}$ are 1 , meaning that the measurements of the other columns of coefficient matrix are with the same precision. The weight matrixes $P_{X}$ and $P_{Y}$ are determined by the precision of each point, namely, weight 
matrixes $P_{X}$ and $P_{Y}$ are determined by point intensity. $E_{i}(i=1,2, \cdots, n)$ denotes the intensity of point $i$.Note here $Q_{Y}$ is regular, but $Q_{0}$ (and thus $Q_{A}$ ) is not.

\section{B. Methods and its precision estimation}

From the formulas derived in the second part of this paper, the proposed method can be designed. The steps of the method are as follows:

1st step: define $P_{0} 、 P_{X}$ and $P_{Y}$ based on the intensity value of each point according to Eq.(15), then generate $Q_{0} 、 Q_{X} 、 Q_{Y}$ and $Q_{A}$ according to Eq.(11) and Eq. (12).

2nd step: $[N, c]=A^{T} P_{Y}[A, Y], \hat{X}^{(0)}=N^{-1} c$

3rd step: calculate $\hat{\lambda}^{(i)} 、 \hat{v}^{(i)}$ and $\hat{X}^{(i+1)}$

$$
\left.\begin{array}{c}
\hat{\lambda}^{(i)}=\left(Q_{Y}+\left(\left(X^{(i) T} Q_{0} X^{(i)}\right) \cdot Q_{X}\right)^{-1}\left(Y-A \hat{X}^{(i)}\right)\right. \\
\hat{v}(i)=\left(\hat{\lambda}^{(i)}\right)^{T} Q_{X} \hat{\lambda}^{(i)} \\
\hat{X}^{(i+1)}=\left(A^{T}\left(Q_{Y}+\left(\hat{X}^{(i)}\right)^{T} Q_{0} \hat{X}^{(i)} Q_{X}\right)^{-1} A-\hat{v}^{(i)} Q_{0}\right)^{-1} \\
\bullet\left(A^{T}\left(Q_{Y}+\left(\hat{X}^{(i)}\right)^{T} Q_{0} \hat{X}^{(i)} Q_{X}\right)^{-1} Y\right)
\end{array}\right\}
$$

4th step: Repeat 3rd step until $\left\|\hat{X}^{(i+1)}-\hat{X}^{(i)}\right\|<\varepsilon$ (for a given threshold $\varepsilon$ )

5th step: compute $\sigma_{0}^{2}$ according to Eq. (14).

\section{EXAMPLE ANALYSIS}

In order to verify the proposed method of WTLS, three different material samples were selected, including the targets of defined reflectivity (Lambertian reflector, reflectivity 55\%), wooden plate, and concrete plate, which had different prosperities in homogeneity and flatness. The defined reflectivity target has the best prosperities in homogeneity and flatness, wooden plate takes the second place, and ordinary concrete plate has the worst prosperities. To wooden plate and ordinary concrete plate, their planarity was not quantified, but visual inspection indicated that the precision better than $\pm 1 \mathrm{~mm}$. These samples were scanned with a ScanStation C10 terrestrial laser scanner from different positions and thus planar point clouds were obtained. The scanned point clouds of three samples are shown in Fig.1.

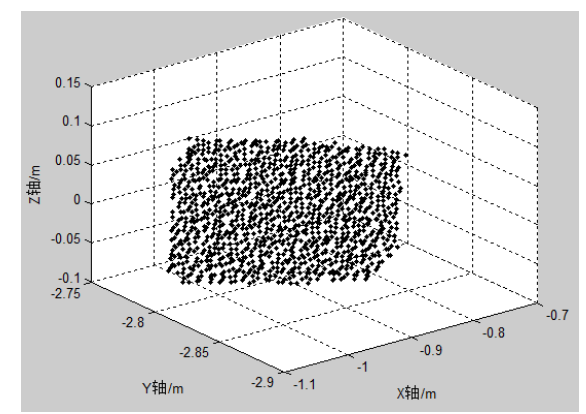

(a) Point clouds of defined target

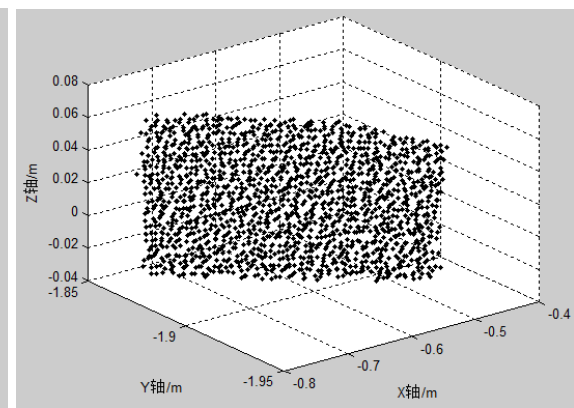

(c) Point clouds of concrete plate

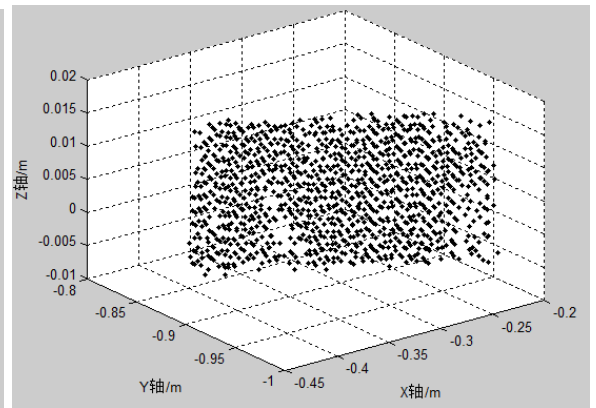

(b) Point clouds of wooden plate

Figure.1 Point clouds of the selected samples

According to the sample data, the form of plane equation could be determined [4]. Based on the plane equation and the considered error factors, surveying adjustment model could be established. At last, three methods, namely, LS method, TLS method and the proposed WTLS method were used for fitting plane and some parameters can be obtained. Except for three planar parameters, $a 、 b$ and $c$, mean square error of unit weight $\sigma_{0}$, and other two parameters $\max \left(d_{i}\right)$ and the root mean squared error of the residuals (RMSE $\left.\sigma_{P}\right)$ were calculated. According to Eq. (17), $d_{i}$ is the normal distance to the fitted surface, which was calculated and the maximum value $\left(\max \left(d_{i}\right)\right)$ was recorded.

$$
d_{i}=\frac{\left|a X_{i}+b Y_{i}-Z_{i}+c\right|}{\sqrt{a^{2}+b^{2}+1}}
$$

$\operatorname{RMSE}\left(\sigma_{P}\right)$, which is an indication of the precision of the residuals based on the fitted plane parameters and the sample observation, was computed as de,scribed in Eq. (18).

$$
\sigma_{P}^{2}=\frac{\sum_{i=1}^{n} d_{i}^{2}}{n}
$$

Our computations were carried out in Matlab. Through a Matlab program, the related parameters obtained by using three methods are shown in table 1.

To the point clouds data from samples with better properties, such as defined reflectivity target, wooden plate, the values of the related parameters calculated with WTLS (the proposed method), such as $\sigma_{0}, \sigma_{P} \max \left(d_{i}\right)$,are better than those calculated with LS and TLS. The two samples have better homogeneity. Due to the different incidence angle of each point on a plane, the intensity value and positional accuracy of each point are different. The point intensity value can be an index of its positional precision. So, as we expected 
the proposed WTLS method is the optimal method, and it is effective for point clouds data with better quality.

To the concrete sample, the proposed method is invalid for its point clouds data. The related parameters calculated with the proposed method is worse than that calculated with TLS, and TLS method seems to be the suitable method for plane fitting. This is because of the complexity of the concrete sample. Concrete sample has poor properties in homogeneity and flatness, which means that there is much noise in its point clouds data. The differences existing in point intensity is caused not only by incidence angle, but also by different materials. So the point intensity value cannot be an index of its positional precision, and we can not use point intensity as its fitting weight. As for TLS method, the assumption that all variances are equal weight avoids the wrong determination in fitting weight. So, to the plane point clouds with large noise, TLS is the most suitable method for plane fitting.

TABLE I. FITTING RESULTS OF PLANE SAMPLES

\begin{tabular}{|c|c|c|c|c|c|c|c|}
\hline \multirow{2}{*}{ Samples } & \multirow{2}{*}{$\begin{array}{r}\text { Met } \\
\text { hods }\end{array}$} & \multicolumn{6}{|c|}{ Calculated related parameters } \\
\hline & & $a$ & $b$ & $c$ & $\sigma_{0}$ & $\sigma_{P}(\mathbf{m m})$ & $\max \left(d_{i}\right)$ \\
\hline \multirow{3}{*}{ Defined target } & LS & 3.5998 & -12.5929 & -39.0822 & 0.044519 & 3.3 & 8.1 \\
\hline & TLS & -15.2431 & -54.7129 & -169.4884 & 0.00061862 & 2.6 & 5.6 \\
\hline & WTLS & -14.913 & -53.2312 & -164.9783 & 0.0005266 & 1.6 & 3.2 \\
\hline \multirow{3}{*}{ wooden plate } & $\mathrm{LS}$ & -0.5302 & -2.2781 & -4.641 & 0.011042 & 4.3 & 8.7 \\
\hline & TLS & -6.2742 & -27.3568 & -55.8717 & 0.00063164 & 2.9 & 5.5 \\
\hline & WTLS & -50.9784 & -221.1237 & -451.8794 & 0.000158968 & 1.4 & 3.7 \\
\hline \multirow{3}{*}{ concrete plate } & LS & -0.23045 & -0.58919 & -0.61775 & 0.0062724 & 5.2 & 9.6 \\
\hline & TLS & -11.0108 & -29.2097 & -30.8396 & 0.00099425 & 3.3 & 6.4 \\
\hline & WTLS & -278.3229 & -739.316 & -780.5754 & 0.0014268 & 3.8 & 7.0 \\
\hline
\end{tabular}

\section{CONCLUSION}

Plane fitting is an important task for creating real 3D modeling from point clouds, and better application can be ensured by improving the fitting plane accuracy. Based on the effect of point intensity on its positional accuracy, a weight total least square method is proposed for plane fitting. From the analysis of this proposed WTSL method with three kinds of sample point cloud data, we studied the applicability of the proposed WTSL method. To the point clouds of the object with better properties in homogeneity and flatness, the proposed method is obviously effective for plane fitting. While to the point clouds of the object with worse properties, because of the complexity of the material, the intensity value of each point cannot denote its positional precision, and the proposed method is invalid for plane fitting, and TLS method is the rather better method.

\section{ACKNOWLEDGMENT}

This work is supported by National Nature Science Foundation (41174002) and Key Laboratory of Precise Engineering and Industry surveying, National Administration of Surveying, Mapping and Geoinformation (PF2011-13).

\section{REFERENCES}

[1] GH. Golub, and Ch. van Loan, "An Analysis of the Total Least Squares Problem," SIAM Journal on Numerical Analysis, vol.17, pp.883-893, 1980.

[2] I. Markovsky, M. Rastello, A. Premoli, A. Kukush, and S. V. Huffel, "The element-wise weighted total least-squares problem," Comput Statist Data Anal, vol.50, pp. 181-209, 2006.

[3] B. Schaffrin, and A. Wieser, "On weighted total least-square adjustment for linear regression," Journal of Geodesy, vol. 82, pp. 415-421, 2008.

[4] A. Bucksch, R. Lindenbergh, and J. V. Ree, Error budge of terrestrial laser scanning: influence of the incidence angle on the scan quality. Russia: In Proceedings GeoSiberia, Novosibirsk, 2007.

[5] J. X. Wei, and K. M. Ji, Industrial measurement fitting. Beijing: Surveying and Mapping Press, 2008. 